\title{
Non-Synaptic Mechanisms of Urinary Bubble Management
}

\section{Berdichevsky VB and Berdichevsky BA* \\ Urology Clinic of Tyumen Medical University, Russia}

*Corresponding author: Berdichevsky BA, Urology Clinic of Tyumen Medical

University, Russia, Email: doktor_bba@mail.ru

\section{Mini Review \\ Volume 3 Issue 3}

Received Date: July 27, 2018

Published Date: August 03, 2018

\section{Abstract}

Scientific works of recent years have shown that neurogenic and non-neurogenic hyperactivity of the bladder is largely a consequence of detrusor ischemia, which is accompanied by structural and functional rearrangement of myocyte cell membranes. Against this background, proteins endogenous activators of contractility of myocytes, which are not neurotransmitters, contacting with extra-synaptic receptors, initiate spasm and clinically manifests idiopathic hyperactivity of the bladder. With further increase in ischemia, the membranes become even stiffer. The phase of hypoactivity or detrucrality of detrusor is coming. The bladder is outside the zone of neurohumoral management, which is clinically manifested by a drop in the capacity of the bladder and an increase in detrusor pressure.

Keywords: Membranes; Relaxation; Emptying; Detrusor

\section{Introduction}

Most of the physiology of urinary excretion is associated with the process of accumulation of urine, which occurs in conditions of moderate sympathicotonia. A conscious urge to urinate and the process of emptying the bladder proceeds against the background of parasympathicotonia. Aceticholine in synapses interacting with cholinergic receptors activates ATPase, which initiates an energy-dependent process of rapid transition of $\mathrm{Na}+$ ions from the extracellular space to the myocyte. The cell in the process of depolarization acquires a positive charge, passing from the state of relaxation to spasm, which ensures the detrusor's exhaustive contractile ability. The work of the parasympathetic synapse is controlled by intrasynaptic (true) cholinesterase. All surpluses of acetylcholine synthesized by nerve endings are neutralized by this enzyme. However, under certain conditions, part of the acetylcholine enters the bloodstream. To do this, the body provides for serum (false) cholinesterase, which neutralizes this neurotransmitter in the vascular bed. At the same time, membranes of red blood cells circulating in the blood can also fix and deposit part of acetylcholine on their surface [1-2].

Numerous scientific works of recent years have shown that neurogenic and non-neurogenic hyperactivity of the bladder is largely a consequence of detrusor ischemia. At the same time, endothelial dysfunction, as a result of impaired muscle nutrition of the bladder, provokes interstitial macrophages to the synthesis of interleukins and tumor necrosis factor-alpha, which in turn stimulates detrusor production, the nerve growth factor in myocytes. Simultaneously, the synthesis of the C-reactive protein, fibrinogen with the appearance of its degradation products, fibrinopeptide A and B is increased in the liver. Together, the products of activation of immune processes and intravascular coagulation are a group of endogenous proteins of myocyte contractility activators capable of activating the detrusor's contractile ability without being synaptic [3]. 
Ischemia of the detrusor is simultaneously accompanied by structural and functional reconstruction of the cellular membranes of the myocyte. In the membranes, the cholesterol content increases and the level of total phospholipids decreases. Oxygen radicals increase the gesture of cytothelium and disrupt the adequate membrane-recognition process. Failure or incomplete neuroreception in these conditions leads to an additional release of free oxygen radicals and further disruption of the fluidity of the lipid bilayer of the myocyte. The process acquires a self-reproducing character [4].

Against this background, proteins endogenous activators of contractility of myocytes that are not neurotransmitters, contacting polyvalent extra-synaptic receptors, initiate phosphorylation of calcium channels with an increase in the current of calcium ions from the intercellular space to the cell, which is accompanied by its spasm and clinically manifested by the so-called idiopathic or independent, but in fact extra-naptic (humoral) hyperactivity of the bladder. With further increase or prolongation of the duration of free radical aggression, the membranes become even more rigid. The mechanism of cell saturation with calcium ions loses its effectiveness. The phase of hypoactivity or detrucrality of detrusor is coming. Urinary puzyr is outside the zone of neurohumoral management, which is clinically manifested by a decrease or disappearance of urge, a decrease in the capacity of the bladder to contract, a decrease in the maximum flow rate of urine, and an increase in detrusor pressure [5].

\section{References}

1. Fry CH, Sahai A, Vahabi B, Kanai AJ, Birder LA (2014) What is the role for biomarkers for lower urinary tract disorders? ICI-RS 2013. Neurourol Urodyn 33(5): 602-605.

2. Lamb K, Gebhart GF, Bielefeldt K (2004) Increased nerve growth factor expression triggers bladder overactivity. J Pain 5(3): 150-156.

3. Seeger H, Wallwiener DA, Mueck FO (2009) Effects of drospirenone on cardiovascular markers in human aortic endothelial cells. Climacteric 12(1): 80-87.

4. Seth JH, Sahai A, Khan MS, van der Aa F, de Ridder D, et al. (2013) Nerve growth factor (NGF): a potential urinary biomarker for overactive bladder syndrome (OAB)? BJU Int 111(3): 372-380.

5. Osman N, Capple C, Abrams H. et al. (2014) Detrusor underactivity and underactivity blader: a new clinical entini. A revier of current terminology? definitions, epidemiology, aetiology and diagnosis. Eur Urol 65(2): 389-398.

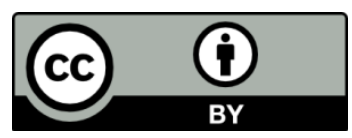

